\title{
Mediación y Geometría Dinámica: una alternativa para involucrar a los estudiantes en la actividad demostrativa en geometría ${ }^{1}$
}

\author{
Dynamic Measurement and Geometry: An Alternative to \\ engage students in the activity demonstrative geometry \\ Medição Dinâmica e Geometria: uma alternativa para envolver \\ os alunos na atividade geometria demonstrativa
}

Recibido: mayo 2013

Aceptado: agosto 2013
Cristina Mejía ${ }^{2}$

Oscar Molina ${ }^{3}$

\begin{abstract}
Resumen
En este taller se presenta parte de una secuencia didáctica con la que se pretende favorecer la actividad demostrativa en una clase de geometría de grado octavo. Tal secuencia, fue diseñada con base en la aproximación metodológica para la enseñanza del grupo Æ•G. Con el taller se pretende ejemplificar cómo podría ser el rol del profesor cunado emplea artefactos bajo la Teoría la Mediación Semiótica propuesta por Mariotti (2009). En este caso el artefacto empleado es Geogebra, un software de geometría dinámica (SGD). La secuencia hace parte de mi trabajo de grado para optar por el título de maestría en Docencia de las Matemáticas y lo que se pretende abordar en el Taller es resultado parcial del mismo.
\end{abstract}

Palabras clave: Matemáticas escolares; Geometría Dinámica; Metodología de trabajo en el aula; Secuencia Didáctica; Mediación Semiótica; Aprendizaje; Procesos cognitivos; procesos de justificación; Actividad demostrativa.

\begin{abstract}
This workshop presents part of a teaching sequence with which the activity is to encourage class demonstration in eighth grade geometry. This sequence was designed based on the methodological approach for teaching • Æ group G. With the workshop is to illustrate how could be the role of teacher artifacts Cunado used under Semiotic Mediation Theory proposed by Mariotti (2009). In this case the device used is Geogebra, dynamic geometry software (SGD). The sequence is part of my grade to qualify for the master's degree in Teaching of Mathematics and intended to be addressed in the workshop is the result in part.
\end{abstract}

1 Artículo de Investiigación.

2 Estudiante de Maestría Universidad Pedagógica Nacional. The English School. Bogotá, Colombia. Contacto: mdma_cmejia758@ pedagogica.edu.co

3 Universidad Pedagógica Nacional. Bogotá, Colombia. Contacto: ojmolina@pedagogica.edu.co 
Keywords: school mathematics; Dynamic Geometry; Methodology of work in the classroom didactic sequence; Semiotic Mediation, Learning, Cognitive processes, processes of justification; Activity demonstrative.

\section{Resumo}

Esta oficina apresenta parte de uma seqüência de ensino com as quais a atividade é incentivar a demonstração de classe na geometria oitava série. Esta seqüência foi projetado com base na abordagem metodológica para o ensino - Æ grupo G. Com a oficina é para ilustrar como poderia ser o papel do professor artefatos Cunado usado sob Theory mediação semiótica proposto por Mariotti (2009). Neste caso, o dispositivo é utilizado Geogebra, geometria dinâmica (BRL). A seqüência é parte da minha classe para se qualificar para o mestrado em Ensino de Matemática e destinados a serem abordados na oficina é o resultado, em parte.

Palavras-chave: matemática escolar; geometria dinâmica, metodologia de trabalho na seqüência didática em sala de aula; mediação semiótica, aprender, processos cognitivos, os processos de justificação; atividade demonstrativa.

\section{Introducción}

En mis clases de Geometría como estudiante, las experiencias que tuve en relación con el sentido de la demostración fueron muy pobres y nunca comprendí claramente que era lo que el profesor pretendía, más allá de memorizar definiciones y repetir demostraciones que ya estaban en los libros. Ahora como aspirante a Magister de la Universidad Pedagógica Nacional, considero que las experiencias en torno a el sentido de la demostración que deben tener mis estudiantes pueden ser muy ricas y que mi rol como profesora realmente puede ayudar a que sus significados personales evolucionen, especialmente cuando en el proceso de enseñanza se utilizan deliberadamente artefactos como un SGD. En los Estándares Básicos de Competencias Matemáticas (2003) se pone de manifiesto que en la escuela se deben proponer situaciones ricas en las que los estudiantes comprendan el papel de la demostración en las matemáticas, y resaltan que se deben aprovechar los recursos didácticos como los software especializados; se reconocen estos recursos como mediadores en el proceso de enseñanza aprendizaje y como elementos integradores de distintos pensamientos que además motivarían a los estudiantes por cuanto les presenta entornos dinámicos.

Atendiendo a lo anterior, en este taller, presento parte de una secuencia didáctica con la que se pretende favorecer el involucramiento de los estudiantes en una actividad demostrativa ${ }^{4}$ y describir cuál podría ser el rol del profesor cuando emplea artefactos como SGD con el propósito de que significados de los estudiantes evolucionen.. Específicamente, el diseño de secuencia didáctica está fundamentado en la aproximación metodológica para la enseñanza del grupo Aprendizaje y Enseñanza de la Geometría Æ•G de la Universidad Pedagógica Nacional, y el análisis del rol del profesor se realiza teniendo como referente la Teoría de la Mediación Semiótica (TMS) propuesta por Mariotti (2009).

\section{Marco teórico}

La TMS basada en el enfoque Vigotskiano (Mariotti, 2009), proporciona el marco teórico para plantear cuál podría ser el rol del profesor. Según este enfoque, el conocimiento es una consecuencia de

4 Actividad conformada por dos procesos, el de conjeturación (cuyo producto es una conjetura) y el de justificación (cuyo producto es la explicación, prueba o demostración del enunciado conjeturado) 
actividades instrumentadas que emergen y evolucionan dentro de la interacción social; en un contexto educativo, el rol del profesor es vital en dicha interacción. Esta teoría reconoce que la enseñanza y el aprendizaje pueden ser vistos como la evolución de signos de los estudiantes (e.g., sus gestos, su producción oral o escrita, o una construcción realizada empleando un software de geometría dinámica). Los signos de los estudiantes, que surgen mediante el uso intencionado de un artefacto, pueden ilustrar sus significados personales, y con la mediación del profesor, pueden evolucionar a signos matemáticos asociados a significados matemáticos (una definición o un teorema por ejemplo). Mariotti (2009) sostiene que reconocer el potencial semiótico de un artefacto $^{3}$ en términos de los significados personales y de significados matemáticos permite al profesor, quien es el experto, emplear los artefactos como herramientas de mediación semiótica, y posibilita que los estudiantes conecten sus significados personales, generados por el uso del artefacto, con significados matemáticos reconocidos por dicho experto.

Los signos producidos por los estudiantes pueden estar muy pegados al artefacto, es decir, ser producto del uso del artefacto para el abordaje de una tarea específica, o ser producto de la reconstrucción de un contexto relacionado con la actividad desarrollada con el artefacto; estos signos para la TMS son los "signos del artefacto" que se espera evolucionen a "signos matemáticos" (i.e., aquellos que están asociados a la teoría matemática misma). Los "signos pivote" son los signos que evidencian conexión entre el contexto del artefacto y el contexto matemático o los que facilitan la transición de un contexto al otro. La producción de signos está fuertemente ligada entonces, a las acciones que fueron propuestas para ser desarrolladas con el artefacto (Drijvers, Kieran \& Mariotti, 2010).

La TMS propone el diseño e implementación de un ciclo didáctico, constructo que se utiliza para referirse a una secuencia de actividades que permite desarrollar los componentes del proceso semiótico (Mariotti, 2009). En términos generales el ciclo didáctico consta de una secuencia enmarcada en tres momentos: Se propone una tarea a los estudiantes en la que se debe hacer uso del artefacto y se realiza el desarrollo de la tarea empleando los artefactos, por parte de los estudiantes. Se realiza una etapa de producción individual de signos como solución a la tarea en la que el profesor, a partir de los signos producidos por los estudiantes, se orienta la discusión hacia una discusión matemática. A partir de la producción colectiva de signos, la acción intencionada del profesor propicia la evolución de los signos producidos por los estudiantes

Para el trabajo de grado (y claro, para el desarrollo de este taller), como ciclo didáctico se utilizó una secuencia fundamentada en la aproximación metodológica para la enseñanza propuesta por el grupo de investigación Æ•G. Esta aproximación consta de: 1. Resolver un problema de conjeturació ${ }^{5}$ en grupos pequeños de estudiantes con el apoyo de un SGD. 2. Reportar dicho proceso de solución y como consecuencia de ello, construir conjeturas. 3. Retomar, por parte del profesor, el trabajo con el artefacto para permitir una puesta en común de los signos que emergieron al resolver la tarea. 4. Identificar, por parte del profesor, los signos que emergieron bien sea del artefacto, personales, pivote o matemáticos. 5. Emplear, por parte de todos los miembros de la clase, esos signos para favorecer la apropiación del discurso matemático (lenguaje matemático, conjeturas adecuadas, estructura de los enunciados, etc.). 6. Emplear, por parte del profesor, los signos que considera matemáticos, para hacer evolucionar los signos de los estudiantes y negociar los significados.

El propósito de este ciclo es producir un teorema y explicitar el sistema teórico local asociado a este teorema.

5 La relación semiótica doble en que por una parte, los significados personales están relacionados con el uso del artefacto, en particular en relación con el propósito de cumplir la tarea, y por otra parte los significados matemáticos pueden estar relacionados con el artefacto y su uso se llamará el potencial semiótico de un artefacto (Bartolini-Bussi \& Mariotti, 2008).

4 Los problemas abiertos de conjeturación exigen formular una conjetura expresada como condicional; la parte de ésta que se debe buscar (antecedente o consecuente) depende de la información que aporta el problema y la pregunta que se propone. 


\section{Descripción de las tareas que serán propuestas}

Inicialmente se plantea el siguiente problema al que se llamará PROBLEMA CUMBRE.

EN GEOGEBRA: Si A, B y C son tres puntos no colineales en el mismo plano y el punto $\mathrm{A}^{\prime}$ es la simetría axial de $\mathrm{A}$ con respecto a la recta $\mathrm{BC}$, ¿qué puede decir del punto $A^{\prime}$ ?

1. Represente la situación usando el SGD.

2. Explore la construcción.

3. Escriba su afirmación en forma de condicional (no la justifique).

Si (lo que se construyó o está dado) entonces (lo que se descubrió)

4. Provea los elementos que emplearía al realizar una justificación de su afirmación

El propósito de proponer esta actividad en este momento es que los participantes identifiquen qué objetos geométricos deberían estar presentes en su solución. No se pretende que se exponga una solución formal del problema aún.

Se espera que los participantes lleguen a: Si A, B y $\mathrm{C}$ son tres puntos no colineales en el mismo plano y el punto $\mathrm{A}^{\prime}$ es la simetría axial de A con respecto a la recta $\mathrm{BC}$, entonces $\mathrm{A}^{\prime}$ es un punto de la circunferencia con centro en $\mathrm{B}$ y radio $\mathrm{AB}$. Tal como se ilustra en las figuras de la derecha que pueden ser unos de los signos producidos por los estudiantes.

Se espera que los elementos suficientes para proveer la justificación dados por los asistentes al taller sean los siguientes: definición de circunferencia, definición de simetría axial y definición de mediatriz (entre otros).

Realizado lo anterior, se les presentará a los participantes una serie de problemas que se podría proponer a los estudiantes tendiente a construir de manera colectiva en la comunidad de clase, todos los elementos teóricos para resolver dicho problema. Con cada una de tales problemas se establecerá el rol del profesor como mediador, y se buscará que los significados de los participantes evolucionen, tal como podría hacerse en un aula de clase con estudiantes en edad escolar. Cada problema en su solución propicia la producción de signos por parte de los estudiantes, y son estos signos con los que el profesor puede hacer mediación usándolos como pivote, matemáticos o como signos del artefacto.

Esas tareas son las que puede ver en el Anexo 1.

\section{Conclusiones}

Efectivamente este tipo de actividades intencionadas en el aula, propicia la mediación del profesor, de manera tal que el andamiaje realizado por el profesor, permite potenciar el uso de los artefactos.

El profesor como agente experto en una comunidad de clase, puede emplear los artefactos para lograr que los signos personales emergentes, evolucionen a signos matemáticos, y que esto se vea de manera latente en las sesiones de clase, tal como lo sostiene la TMS.

La aproximación metodológica para la enseñanza del grupo Aprendizaje y Enseñanza de la Geometría Æ•G de la Universidad Pedagógica Nacional, es una aproximación efectiva para favorecer la actividad demostrativa en las aulas de clase, y los estudiantes de secundaria pueden desarrollar dicha actividad.

\section{Referencias}

Bartolini-Bussi, M., \& Mariotti, M. (2008). Semiotic mediation in the mathematics classroom: Artifacts and signs after a Vygotskian perspective. Handbook of international research in mathematics education.

5 Archivo de Geogebra, con un punto A y su simetría axial respecto a una recta. 
Drijvers, P., Kieran, C., \& Mariotti, M.-A. (2010). Integrating Technology into Mathematics Education: Theoretical Perspectives. En C. Hoyles, \& J.-B. Lagrange, Mathematics Education and Technology-Rethinking the Terrain. The 17th ICMI Study (Vol. 13, págs. 89-132). London.

Ministerio de Educación Nacional (2003). Estándares básicos de calidad.
Mariotti, M. (2009). Artifacts and signs after a Vygotskian perspective: the role of the teacher. ZDM Mathematics Eduaction, 427-440.

Samper, C., Perry, P., Camargo, L., \& Molina, O. (2011). Capítulo 1: Innovación en un aula de geometría de nivel universitario.

ANEXO 1

TAREA 1 HECHO GEOMÉTRICO:

Si $\square$ A y $\square$ B forman un par lineal y $\square \mathrm{A} \square \square \mathrm{B}$, ¿qué pueden decir de $\square \mathrm{A}$ y $\square \mathrm{B}$ ?

1. Representen la situación usando el SGD.

2. Exploren la construcción.

3. Escriban su afirmación en forma de condicional

Si (lo que se construyó o está dado) entonces (lo que se descubrió)

Provean los elementos que emplearían al realizar una justificación de su afirmación

TAREA 2 DEFINICIÓN parte a:

Copien en sus computadores el archivo GEOMETRÍA $1^{1}$.

1. Exploren la construcción.

2. Activen la herramienta rastro, ¿Qué afirmaciones pueden realizar?

3. Escriba las afirmaciones que puede hacer a partir de su exploración

TAREA 3 DEFINICIÓN parte b:

(Tarea para ser desarrollada con toda la clase, recogiendo, comparando y discutiendo la tarea anterior). En la Tarea 2 las afirmaciones que se hicieron, describen la simetría axial de un punto con respecto a una recta. Escriban entonces la definición de Simetría Axial del punto A, empleando las afirmaciones realizadas en la tarea 2.

TAREA 4 HECHO GEOMÉTRICO:

Dados los puntos A y B, ¿qué condiciones cumplen los puntos que equidistan de A y de B?

1. Realicen la construcción en el SGD

2. Construyan un punto que equidiste de $\mathrm{A}$ y de B

3. Socialicen qué punto construyeron.

4. Escriban su conjetura en forma de condicional.

5. Provean los elementos que emplearían para realizar la justificación de su conjetura.

Fuente: Elaboración propia. 